Seek durabi I ity for CoCrTa per pendi cul ar flexi bl e di sks

\begin{tabular}{|l|l|}
\hline 著者 & $\begin{array}{l}\text { Tagami Kat sumi chi , Takeda Takayuki, Ar ai } \\
\text { Toyoko, Hayashi da H r oshi }\end{array}$ \\
\hline $\begin{array}{l}\text { j our nal or } \\
\text { publ i cat i on t i t l e }\end{array}$ & I EEE Tr ansact i ons on Magnet i cs \\
\hline vol une & 26 \\
\hline number & 5 \\
\hline page range & 2694 2696 \\
\hline year & $1990-09-01$ \\
\hline URL & ht t p: //hdl . handl e. net /2297/17572 \\
\hline
\end{tabular}




\title{
SEEK DURABILITY FOR CoCr'Ta PERPENDICULAR FLEXIBLE DISKS
}

\author{
Katsumichi Tagami, Takayuki Takeda, Toyoko Arai and Hiroshi Hayashida
}

NEC Corporation, Miyazaki 4-Chome, Miyamae-ku, Kawasaki 213

Abstract Seek durability for CoCrTa perpendicular flexible disks was studied using a conventional 3.5" double-sided flexible disk drive. The seek durability strongly depends on the CoCrTa film preparation conditions. The durability is increased by adding a Si underlayer for a CoCrTa film. Disk media yield pressures were evaluated with a scratch test using a sapphire needle. A high media yield pressure leads to a high seek durability. Ta addition to $\mathrm{CoCr}$ film increases the media yield pressure. $200 \times 10^{4}$ seek operations were attained for the disk construction :protective layer/CoCrTa film/Si underlayer/base film.

\section{INTRODUCTION}

Perpendicular magnetic recording provides extremely high recording densities [1]. A key factor in putting the CoCr thin film flexible disks into practical use is mechanical durability. As a method to evaluate the mechanical durability, pass wear tests on the same recording track have been widely carried out $[2,3]$. However, it is difficult to evaluate the mechanical durability for head seek operations wherein the head moves from track to track. This paper describes disk mechanical properties to evaluate head seek durability for a CoCrTa perpendicular flexible disk for a double-sided flexible disk drive (FDD).

\section{EXPERIMENT}

Disk preparation CoCrTa films [4] were deposited on polyimide films only or on polyimide films on which underlayers were formed by r.f. sputtering under two sputtering power $(\mathrm{Pw})$ conditions. $\mathrm{Cr}$ and $\mathrm{Ta}$ concentrations in CoCrTa films are 17 at\% and 3 at\%, respectively. $\mathrm{CoCr}(20 \mathrm{at} \% \mathrm{Cr})$ film was also deposited on a polyimide film. $\mathrm{Si}\left(600 \mathrm{~A}\right.$ thick) and $\mathrm{Al}_{2} \mathrm{O}_{3} / \mathrm{CoCr}(500 \mathrm{~A} / 50 \mathrm{~A}$ thick) films were used as the underlayers. The $\mathrm{CoCr}$ underlayer was used as an adhesive layer between the $\mathrm{Al}_{2} \mathrm{O}_{3}$ film and the polyimide film. The magnetic film was coated with a protective layer and lubricant. Table 1 shows the constructions and preparation conditions for the disks. Thicknesses for polyimide film, magnetic film (CoCrTa and $\mathrm{CoCr}$ film)and protective layer were $30 \mu \mathrm{m}, 0.3 \mu \mathrm{m}$ and 0.03 $\mu \mathrm{m}$, respectively. Head seek resistance for the disks were evaluated using a double-sided FDD (600 rpm), which was controlled by a personal computer. The head seek durability was defined by a seek pass number, at which shallow scratches were observed on the disk surface. The head seek operations were carried out between track 20 to track 50 .

\section{Media scratch test and media yield pressure}

Media scratch resistances were measured by a scratch test apparatus using a sapphire needle (point radius $=0.05 \mathrm{~mm})[2,3]$. Figure 1 shows a cross section view of a disk scratched with the needle. Scratch force (SF) and scratch depth (SD) are determined by the disk horizontal yield pressure and the disk perpendicular yield pressure[5]. Horizontal yield pressure $\mathrm{P}_{H}$ and perpendicular yield
pressure $\mathrm{P}_{\mathrm{P}}$ for a disk are given by

$$
\begin{aligned}
& P_{H}=S F / S_{H} \\
& S_{H}=R^{2}(\pi / 2-0.5 \sin 2 \theta-\theta) \\
& P_{P}=L F / S_{P} \\
& S_{P}=\pi^{*} R^{2}(1-\sin \theta) \\
& \theta=\sin ^{-1}(1-S D / R)
\end{aligned}
$$

where $S_{H}$ and $S_{P}$ are the cross sections of scratched media and the needle surface area respectively. LF and $R$ are the needle load force and the needle point radius, respectively. Scratch force SF can be resolved into base film scratch force term $S F_{h}$ and media scratch force term $S_{m}$ for the magnetic film, protective layer and underlayer. Therefore, the scratch
force is given by

$$
\begin{aligned}
& \mathrm{SF}=\mathrm{SF}_{\mathrm{m}}+\mathrm{SF}_{\mathrm{b}} \\
& \mathrm{SF}_{\mathrm{m}}=\mathrm{P}_{\mathrm{Hin}}{ }^{*} \mathrm{~S}_{\mathrm{Hm}} \\
& \mathrm{S}_{\mathrm{H} \mathrm{m}}=\pi^{*} \mathrm{R}^{2} *\left(0.5^{*} \sin 2 \theta^{\prime}+\theta^{\prime}-0.5^{*} \sin 2 \theta-\theta\right) \\
& \theta=\sin ^{-1}(1-\mathrm{SD} / \mathrm{R}) \\
& \theta^{\prime}=\sin ^{-1}\{1-[\mathrm{SD}-\delta] / \mathrm{R}\}
\end{aligned}
$$

where $\mathrm{P}_{\mathrm{Hm}}$ and $\mathrm{S}_{\mathrm{Hm}}$ are horizontal media yield pressure and film cross section, respectively. Perpendicular media yield pressure $\mathrm{P}_{\mathrm{Pm}}$ is given by

$$
\begin{aligned}
& \mathrm{P}_{\mathrm{Pm}}=\left[L F-\mathrm{P}_{\mathrm{Pb}}{ }^{*} \pi^{*} \mathrm{R}^{2 *}(1-\sin \theta)\right] / \mathrm{S}_{\mathrm{Pm}} \\
& \mathrm{S}_{\mathrm{Pm}}=\pi^{*} \mathrm{R}^{2} *\left(\sin \theta^{\prime}-\sin \theta\right)
\end{aligned}
$$

where $S_{P m}$ is needle surface area when the needle is in contact with the scratched film including the magnetic film, protective film and underlayer. $\mathrm{P}_{\mathrm{Pb}}$ is the perpendicular base film yield pressure.

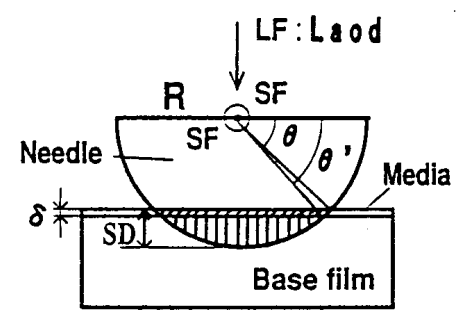

(a)

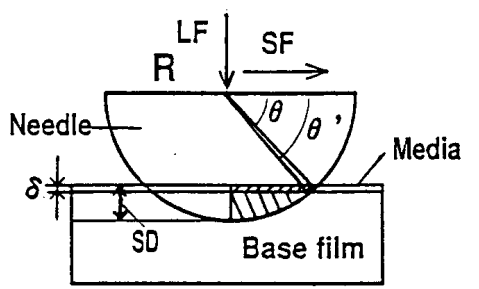

Fig. 1 Disk structure scratched with a needle.

$$
\text { (a) Front view (b) Side view }
$$

\section{Seek durability test}

\section{RESULTS AND DISCUSSION}

Seek durability results for the disks are shown in Table 1. The seek durability strongly depends on disk preparation and film construction. The seek durability pass numbers range from $25 \times 10^{4}$ passes to $200 \times 10^{4}$ passes. In $\mathrm{CoCrTa}$ film disks without the underlayer, the seek resistance for disk $A$ is smaller than that for disk B. Many shallow scratches parallel to the recording track were observed on the surface of disk $A$ at around $40 \times 10^{4}$ passes. However, no scratches were observed on disk $B$ prepared by high sputtering power $\mathrm{Pw}$, up until $90 \times 10^{4}$ passes. The seek durability at $200 \times 10^{4}$ passes was obtained for disk $\mathrm{C}$ with $\mathrm{Si}$ underlayer. The durability value is two times greater than that for disk $\mathrm{B}$. The seek durability for disk $\mathrm{D}$ with $\mathrm{Al}_{2} \mathrm{O}_{3} / \mathrm{Cr}$ underlayer was $25 \times 10^{4}$ passes, but there was no improvement in the seek resistance. However, the seek durability is increased by forming the $\mathrm{Si}$ underlayer on the base film. The seek durability for the disk $\mathrm{E}$ of CoCr media was $20 \times 10^{4}$ passes, which is less than that for CoCrTa 

Table 1. Flexible disk construction and seek durability
pass number.

\begin{tabular}{|c|c|c|c|c|}
\hline Disk & Media & $\begin{array}{c}\text { under } \\
\text { layer }\end{array}$ & $\begin{array}{c}\mathrm{Pw} \\
\left(\mathrm{W} / \mathrm{cm}^{2}\right)\end{array}$ & $\begin{array}{c}\text { durability } \\
\left(\times 10^{4}\right)\end{array}$ \\
\hline $\mathrm{A}$ & $\mathrm{CoCrTa}$ & - & 0.4 & 40 \\
\hline $\mathrm{B}$ & $\mathrm{CoCrTa}$ & - & 0.8 & 90 \\
\hline $\mathrm{C}$ & $\mathrm{CoCrTa}$ & $\mathrm{Al}_{2} \mathrm{O}_{3}$ & 0.8 & 25 \\
\hline $\mathrm{D}$ & $\mathrm{CoCrTa}$ & $\mathrm{Si}$ & 0.8 & 200 \\
\hline $\mathrm{E}$ & $\mathrm{CoCr}$ & - & 0.8 & 20 \\
\hline
\end{tabular}

\section{Scratch test}

$\checkmark$ order to clarify the seek durability differences for test disk eratch resistances were examined. Figure 2 shows disk siratch depths versus scratch needle load force. In the low load force region $(<20 \mathrm{~g})$, scratch depth(SD)/load force(LF) values for all test disks are almost the same, due to the protective layer effect against head scratch force. However, in the greater load force region, SD/LF values differ from disk to disk. The disk seek durability increases with the SD/LF value decrease.

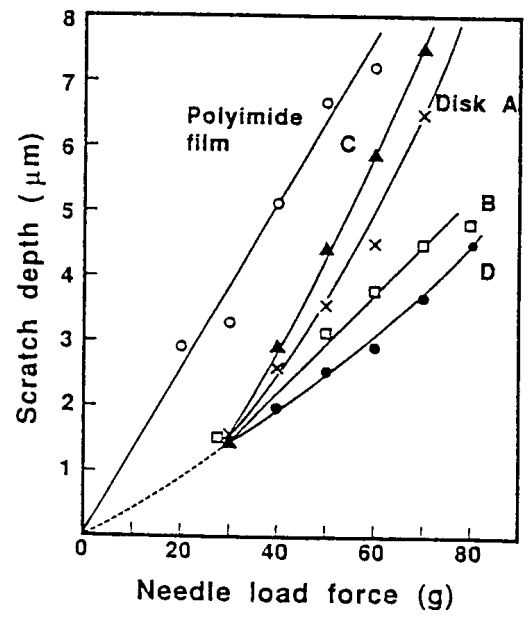

Fig. 2 Disk scratch depths versus scratch needle load force.

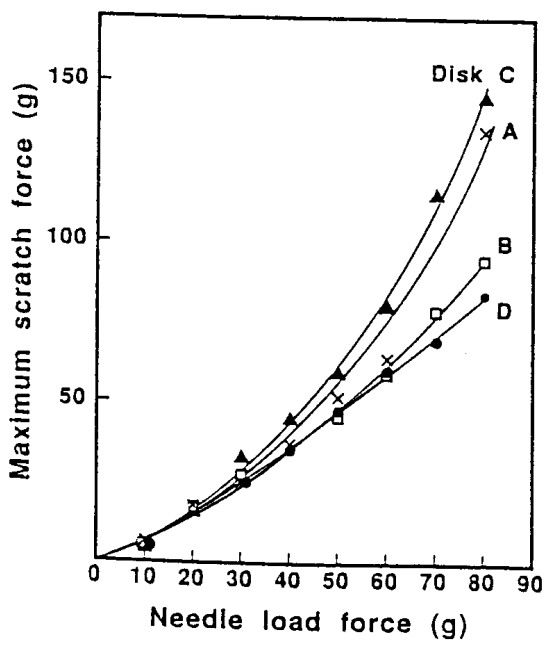

Fig. 3 Maximum scratch force values for test disks versus needle load force.
Figure 3 shows maximum scratch force values for the disks versus needle load force. The scratch force values depend on SD/LF values for individual disk. Disk D with Si underlayer has the lowest scratch force value among the disks. On the other hand, the scratch force for disk $C$ with $\mathrm{Al}_{2} \mathrm{O}_{3} / \mathrm{Cr}$ underlayer is the greatest. Figure 4 shows scratch force variation (amplitude) versus needle load force for the disks. The scratch force variation is due to scratch depth variation. In the 0 to $20 \mathrm{~g}$ load force range, variation amplitudes are almost the same, because there are no scratches on the disk surfaces. However, at $30 \mathrm{~g}$ load force, scratch force variation peaks for disks $A, B$ and $C$ are observed. This is explained by considering that protective layers begin to be broken with scratch needle at around $30 \mathrm{~g}$ load force. In the great load force region $(>30 \mathrm{~g})$, disks $\mathrm{A}$ and $C$ with low seek durabilities have large scratch force variations. On the other hand, disks $B$ and $D$ show a stable scratch force variation and the variation values are also small.

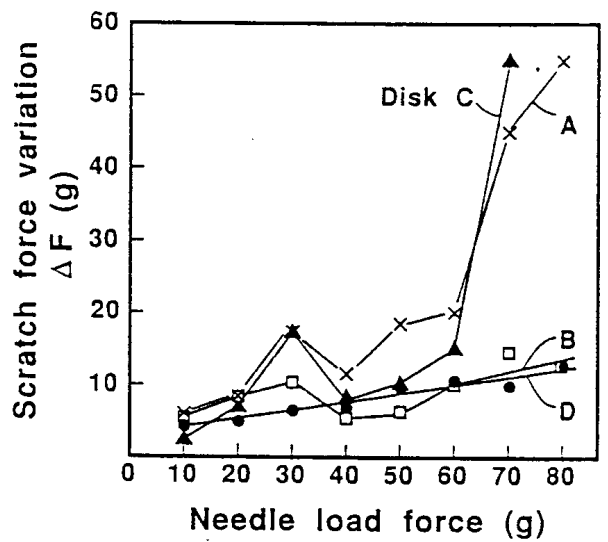

Fig. 4 Scratch force variation (amplitude) versus needle load force for test disks.

\section{Disk media yield pressure}

Scratch force is determined by a horizontal yield pressure for an entire disk media including a base film, an underlayer, a magnetic film and a protective overlayer. Disk horizontal yield pressure, in Eq.(1), is calculated for the test disks. Figure 5 shows the disk horizontal yield pressure versus needle load force. Horizontal yield pressure magnitudes are in the order:

Disk $\mathrm{D}>$ Disk $\mathrm{B}>$ Disk $\mathrm{A}>$ Disk $\mathrm{C}>$ Polyimide film

The yield pressure magnitudes correspond to those for the disk seek durability. Figure 6 shows the disk perpendicular

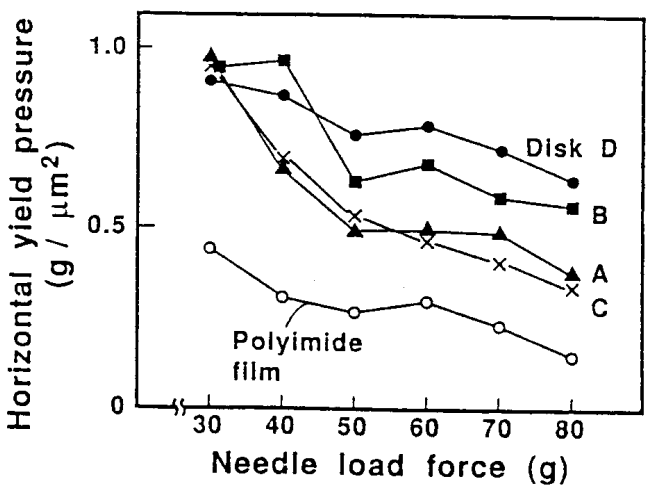

Fig. 5 Disk horizontal yield pressure versus needle load force. 


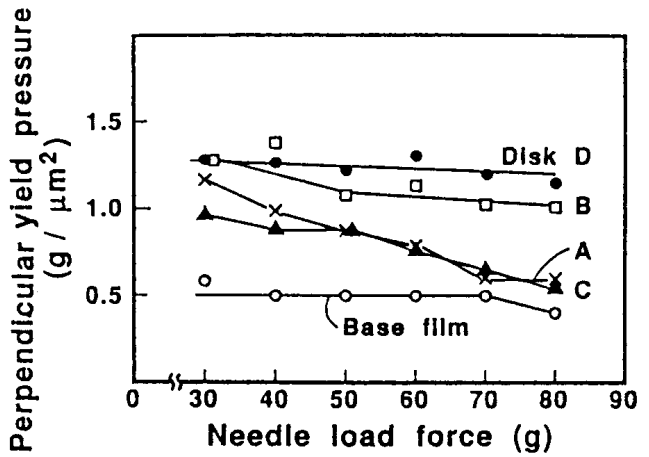

Fig. 6 Disk perpendicular yield pressure versus needle load force.

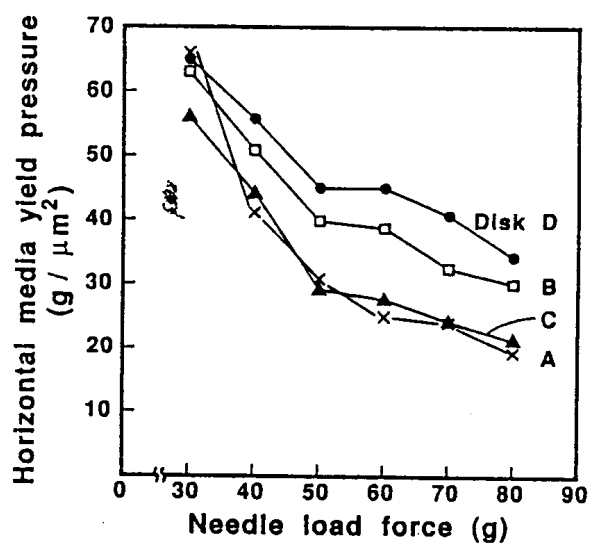

Fig. 7 Media yield pressure versus needle load force.

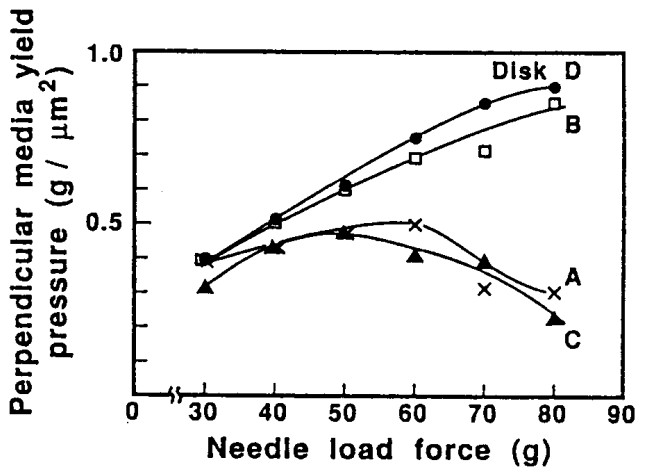

Fig. 8 Media perpendicular yield pressure versus needle load force.

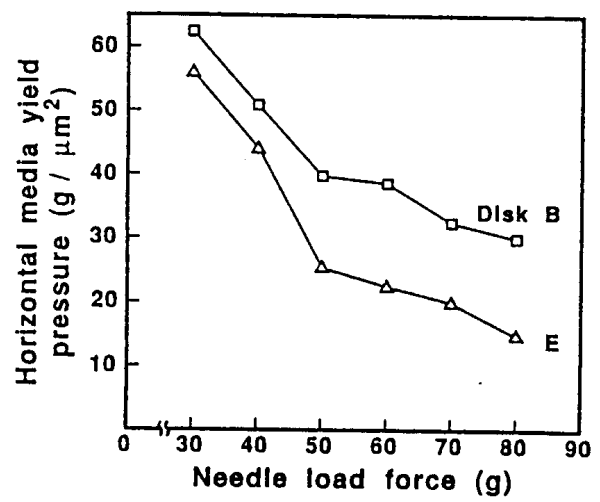

Fig. 9 Horizontal media yield pressure versus needle load force for test disks $B$ and $E$. yield pressure of Eq.(2) versus needle load force. The perpendicular yield pressure magnitude order for the disks is the same as that for the horizontal yield pressure.

A media yield pressure, except for a base film yield pressure, was evaluated by using Eq.(4) and Eq.(5). Figure 7 shows the horizontal media yield pressure versus needle load force. The relation of media yield pressure to needle load force shows in Fig. 5 a tendency similar to that for an entire disk yield pressure. This result shows that the media yield pressure determines the entire disk yield pressure. Figure 8 shows the perpendicular media yield pressure versus needle load force. Disks $B$ and $D$, with high seek durabilities, have high perpendicular media yield pressures. The Si underlayer increases the horizontal and perpendicular media yield pressures. However, disk $\mathrm{C}$. with $\mathrm{Al}_{2} \mathrm{O}_{3}$ underlayer has less seek durability. This is considered to be due to insufficient $\mathrm{Cr}$ film adhesion between the underlayer and the polyimide film. On the other hand, Disk A, prepared under low sputtering power, has half the seek durability for disk $B$ prepared at a high sputtering power. The durability deterioration is due to a lowering of the media yield pressure, especially the perpendicular media yield pressure.

In order to evaluate the effect of adding $\mathrm{Ta}$ to the $\mathrm{CoCr}$ media, the horizontal media yield pressures for disks $B$ and $E$ were examined. Figure 9 shows the horizontal media yield pressure versus needle load force for disks B and $E$ indicating that the horizontal yield pressure for disk $B$ is two times greater than that for disk $\mathrm{E}$ without Ta. The addition of Ta increases the mechanical strength for the $\mathrm{CoCr}$ film [7]. Therefore, a high media yield pressure is needed to obtain a high seek resistance. These results show that mechanical strength of disk medium is a very important factor, which determines seek durability and that a seek durability of $200 \times 10^{4}$ passes can be attained by using appropriate conditions and a hard underlayer.

\section{CONCLUSION}

The seek durability for CoCrTa perpendicular flexible disks was studied using a conventional 3.5" double-sided FDD. The seek durability strongly depends on the CoCrTa film preparation conditions. The durability is strengthen by adding a Si underlayer to a CoCrTa film and by addition of $\mathrm{Ta}$ to a CoCr film. The mechanical strength increase is attributed to an increase in the media yield pressure. A high media yield pressure leads to a high seek durability. $200 \times 10^{4}$ seek operations were attained for the following media construction: protective overlayer/CoCrTa film/Si underlayer/base film.

\section{ACKNOWLEDGMEN'T}

The authors would like to thank Dr. S. Esho and Dr. H. Gokan for their encouragement and Mr. H. Tamai for his discussion.

\section{REFERENCE}

[1] S. Yamamoto, Y. Nakamura, S. Iwasaki, IEEE Trans. MAG- 23, 2070 (1987)

[2] K. Tagami, H. Tamai, H. Hayashida and T. Arai, IEEE Trans. Magn., MAG-24, 2655 (1988)

[3] K. Tagami, H. Tamai, T. Arai and H. Hayashida Journal of The Magnetics Society of Japan Vol.13, Supplement, No. S1, p219 (1989)

[4] H. Tamai, $K$. Tagami and H. Hayashida, IEEE Trans. Magn., MAG-24, 2347 (1988)

[5] Horst Czichos, Tribology, Elsevier scientific publishing company, 1978

[6] Y. Motomura and K. Tagami, IEEE Trans. Magn. MAG-22, 346 (1986)

[7] M. Naoe and M. Matsuoka, J. Apply. Phys., 57(1), 4019 (1985) 